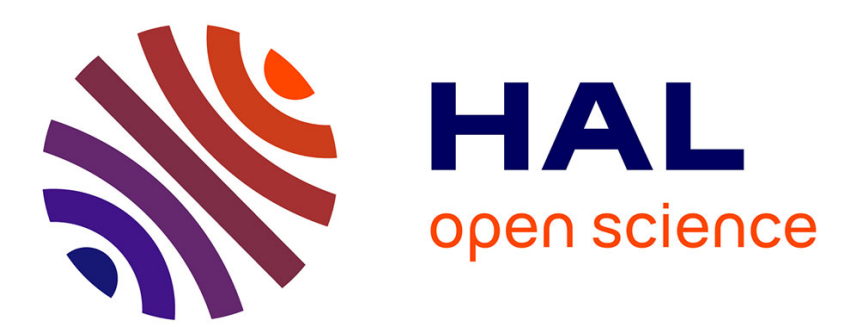

\title{
SPECTRAL CLUSTERING BASED PARCELLATION OF FETAL BRAIN MRI
}

\author{
Antonietta Pepe, Guillaume Auzias, François de Guio, François Rousseau, \\ David Germanaud, Jean-François Mangin, Nadine Girard, Olivier Coulon, \\ Julien Lefèvre
}

\section{To cite this version:}

Antonietta Pepe, Guillaume Auzias, François de Guio, François Rousseau, David Germanaud, et al.. SPECTRAL CLUSTERING BASED PARCELLATION OF FETAL BRAIN MRI. 2015. hal01114989

\author{
HAL Id: hal-01114989 \\ https://hal.science/hal-01114989
}

Preprint submitted on 10 Feb 2015

HAL is a multi-disciplinary open access archive for the deposit and dissemination of scientific research documents, whether they are published or not. The documents may come from teaching and research institutions in France or abroad, or from public or private research centers.
L'archive ouverte pluridisciplinaire HAL, est destinée au dépôt et à la diffusion de documents scientifiques de niveau recherche, publiés ou non, émanant des établissements d'enseignement et de recherche français ou étrangers, des laboratoires publics ou privés. 


\title{
SPECTRAL CLUSTERING BASED PARCELLATION OF FETAL BRAIN MRI
}

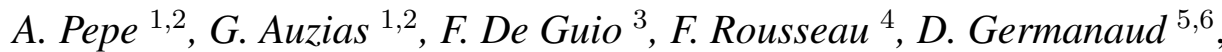 \\ J.-F. Mangin ${ }^{7}$, N. Girard ${ }^{8}$, O. Coulon ${ }^{1,2}$, J. Lefèvre $^{1,2}$ \\ ${ }^{1}$ Institut de Neurosciences de la Timone UMR 7289, Aix Marseille Université , CNRS, Marseille, France \\ ${ }^{2}$ Aix Marseille Université, CNRS, LSIS laboratory, UMR 7296, Marseille, France \\ ${ }^{3}$ Univ Paris Diderot, Sorbonne Paris Cité, UMR-S 1161 INSERM, Paris, France \\ ${ }^{4}$ ICube, Université de Strasbourg/CNRS, UMR 7357, Illkirch, France \\ ${ }^{5}$ CEA, NeuroSpin, UNIACT, F-91191 Gif-sur-Yvette, France \\ ${ }^{6}$ AP-HP, Hôpital Robert Debré, DHU PROTECT, F-75019 Paris, France \\ ${ }^{7}$ CEA, UNATI, Neurospin, Gif-sur-Yvette, France \\ ${ }^{8}$ APHM, CHU Timone, Service de Neuroradiologie, Marseille, France
}

\begin{abstract}
Many neuroimaging studies are based on the idea that there are distinct brain regions that are functionally or microanatomically homogeneous. Obtaining such regions in an automatic way is a challenging task for fetal data due to the lack of strong and consistent anatomical features at the early stages of brain development. In this paper we propose the use of an automatic approach for parcellating fetal cerebral hemispheric surfaces into $K$ regions via spectral clustering. Unlike previous methods, our technique has the crucial advantage of only relying on intrinsic geometrical properties of the cortical surface and thus being unsupervised. Results on a data-set of fetal brain MRI acquired in utero demonstrated a convincing parcellation reproducibility of the cortical surfaces across fetuses with varying gestational ages and folding magnitude.
\end{abstract}

Index Terms - Fetal MRI, spectral clustering, LaplaceBeltrami Operator, brain lobes.

\section{INTRODUCTION}

Brain parcellation techniques aim at dividing the brain into units that are functionally or micro-anatomically homogeneous. Parcellation methods used for adult and post-natal brain studies often rely on the geometry of cortical folds, such as the correspondence between sulcal fundi and boundaries of distinct cortical areas. However, in fetal brains, the folding is still developing and data-driven approaches might lack of consistent anatomical features that are required to ensure the definition of cortical parcels. To study region-specific brain development in fetal data, we would need a parcellation scheme that is independent of cortical folding and its rapid changes over time. A recent parcellation method [1]

This study is funded by the Agence nationale de la recherche (ANR-12JS03-001-01 "MoDeGy"). based on a random sampling of the cortical surface has been proposed with encouraging results for connectivity analysis. Such parcels however lack of anatomical and/or functional meaning in the context of region-based analysis such as e.g. the study of distinct surface growth trajectories across cortical regions. Spectral clustering methods [2] offer an alternative approach for reproducible mesh partitioning which rely only on global geometric shape invariants. In a recent study [3], we have proposed an automatic and unsupervised method based on spectral clustering that parcellates cortical surfaces into regions having striking similarities with brain lobes, a coarser resolution for brain information that allows a better matching of different anatomies [3]. A natural question can then be to establish whether this individual segmentation strategy can be successfully applied to parcellate fetal cortical surfaces into lobes despite the lack of strong and consistent anatomical features at this early stage of brain development. We quantitatively assess the anatomical relevance of our parcellation algorithm by measuring, within each subject, the alignment of parcel boundaries with two early folds manually traced by an expert. We also provide qualitative and quantitative evidence of the good reproducibility of our parcels across individuals at different gestational ages.

\section{METHODS}

\subsection{Parcellation based on spectral clustering}

In this work, a parcellation $f: \mathcal{S} \rightarrow\{1, \ldots, K\}$ of cortical surfaces into $K$ patches is obtained using spectral clustering. Spectral clustering are an effective family of methods that treats the clustering (segmentation) of the data as a graph partitioning problem $[4,5]$. In spectral clustering, a partitioning of the data is inferred from local neighborhood relations (e.g. similarities between data points) in an unsupervised 
manner, that is with no prior information on the data distribution. More specifically, the first (smallest) $K$ eigenvectors of a matrix derived from similarities between data points are used for dimensionality reduction. Once the eigenvalue decomposition problem is solved, a simple clustering algorithm (e.g K-means clustering) can be applied to the new simplified low-dimensional embedding to partition data points into $K$ clusters $[4,5]$. Among the many possible metrics and similarity structures that have been proposed (for a review see [4]), graph laplacians obtained from sparsified similarity matrices have the advantage of providing computational and memory simplifications, and of guaranteeing non-negative eigenvalues.

We use here Fourier modes of the cortical surface $\mathcal{S}$ at a given order as inputs of the spectral clustering algorithm since they reflect the geometry of the original surface, are intrinsic, do not require parameters tuning, and only require a small neighborhood to compute the pairwise similarity between data points [3]. More precisely the Laplace-Beltrami operator (LBO), here denoted as $\Delta_{\mathcal{S}}$, has a basis of orthonormal eigenfunctions $\phi_{0}, \phi_{1}, \ldots$ which satisfies $-\Delta_{\mathcal{S}} \phi_{j}=\lambda_{j} \phi_{j}$, where the set of eigenvalues $\lambda_{0}=0 \leq \lambda_{1} \leq \lambda_{j} \ldots$ is also referred to as spectrum, and the eigenfunctions $\phi_{j}$ are also referred to as Fourier modes [3]. To compute those modes we use the finite element method $[3,6]$. The resulting discretized problem becomes finding a vector $U=\left(u_{i}\right)_{i=1 . .|\mathcal{S}|}$ and a scalar $\lambda$ such that $\mathbf{G} U=\lambda \mathbf{M} U$, with $i$ being one the $|\mathcal{S}|$ vertex indices in mesh $\mathcal{S}$, and $\mathbf{G}$ and $\mathbf{M}$ being the stiffness and mass matrices, respectively (see [6] for details). Since $\mathbf{G}$ and $\mathbf{M}$ are sparse and symmetric positive matrices, the above discretized eigenvalue problem can be solved with sparse eigensolvers such as the Lanczos method [3]. A more detailed introduction to LBO can be found in [7].

\subsection{Parcel matching}

Spectral clustering does not guarantee that anatomically corresponding brain surface patches are assigned to a same label. To achieve consist labelings, the individual parcellation maps obtained by the spectral algorithm are mapped into a common spherical space $\mathbb{S}^{2}$ using a bijective parametrization technique called "HIP-HOP" [8]. Next, a remapping is obtained in this space.

Using HIP-HOP, each individual segmentation map $f_{p}$ of a surface $S_{p}$ is mapped into $\mathbb{S}^{2}$ via the corresponding parametrization $\mathbf{m}_{\mathcal{S}_{p}}: \mathbb{S}_{p} \longrightarrow \mathbb{S}^{2}$ to obtain the projected map $f_{p}{ }^{\prime}=f_{p} \circ \mathbf{m}_{\mathcal{S}_{p}}$. This is done for all the $N$ cortical surfaces included in the study. The resulting projected maps are vectors of length 40962 consisting of integer values ranging from 1 to $K$. A relabeling $\sigma:[1, K] \longrightarrow[1, K]$ of the spherical maps $f_{1}{ }^{\prime}, \ldots, f_{p}{ }^{\prime}, \ldots f_{N}{ }^{\prime}$ is then computed via the Munkres algorithm (also known as Hungarian algorithm) [9], an efficient combinatorial algorithm that tackles the assignment problem in polynomial-time. For two given segmentation maps, an individual $f_{p}{ }^{\prime}$ and a reference $f_{r e f}{ }^{\prime}$ map, a $K \times K$ cost optimizing assignment for $f_{p}{ }^{\prime}$ is calculated based on the following two measures: (1) the Dice coefficients measuring the overlap between each of the $K$ parcel labels in $f_{p}{ }^{\prime}$ with respect to all the others in $f_{\text {ref }}{ }^{\prime}$ (if $K \leq 6)$; (2) the sum of the Euclidean distances between each of the $K$ centers of mass of surface patches projected into the spherical surface (if $K>6$ ). The optimal relabeling $\sigma_{p}$ of $f_{p}{ }^{\prime}$ is the one optimizing the cost. For clarity of notation, in the following we use $f_{p}$ for the native space, $f_{p}{ }^{\prime}$ for its mapped version into $\mathbb{S}^{2}$, and $g_{p}$ for the relabeled map in $\mathbb{S}^{2}$.

The parametrization of the parcellation maps into the common spherical space $\mathbb{S}^{2}$ also provided computational simplifications for the evaluation of the parcellation reproducibility across-subjects which will be described next.

\subsection{Quantitative evaluation}

The reproducibility of the spectral clustering based parcellation of cortical surfaces is assessed quantitatively by computing two measures of parcellation accuracy for various $K$ values.

Individual evaluation. As a first measure, we consider a geometric measure of the accuracy of a parcellation, here denoted as $D_{s}(K)$. It is defined as the average, over all the subjects, of the distances between anatomical relevant landmarks $l_{m}$ in the individual cortical surface $S_{p}$ and the boundaries of the corresponding segmentation map $f_{p}$ (in the native space) for a certain value of $K$

$$
D_{s}(K)=\frac{1}{N} \sum_{p=1}^{N} \frac{1}{\operatorname{Area}\left(S_{p}\right)} \sum_{m=1}^{M} \operatorname{Dist}_{1}\left(l_{m}, f_{p}\right)^{2}
$$

where $\operatorname{Dist}_{1}\left(l_{m}, f_{p}\right)$ denotes the mean Euclidean distance of the points along a landmark $l_{m}$ to the boundary of a segmentation map $f_{p}$. In our experiments, we consider as landmarks $l_{m}$ the points along the Central Sulcus (CS) and the Parietooccipital Sulcus (POS) $(M=2)$ since they have been reported to be among the first sulci appearing in the developing fetal brain [10]. They are also known to define anatomicl boundaries between the fronatl and parietal lobes (CS), and between the occipital and parietal lobes (POS).

Evaluation at the group level. As second measure, we consider the reproducibility $D_{r}(K)$ of a surface parcellation with $K$ parcels derived as the sum over all the subjects (in the spherical space) of pair-to-pair measures $\operatorname{Dist}_{2}\left(g_{p}, g_{q}\right)$ obtained as

$$
\operatorname{Dist}_{2}\left(g_{p}, g_{q}\right)=\frac{1}{K} \sum_{k=1}^{K}\left(1-\operatorname{Dice}\left(g_{p}^{-1}(k), g_{q}^{-1}(k)\right)\right)
$$

where Dice is the usual Dice coefficient between two sets. Notice that $\operatorname{Dist}_{2}\left(g_{p}, g_{q}\right)$ depends on $K$ even if not explicitly mentioned in the notation.

The optimal number of clusters $K$ is then evaluated as the one optimizing the reproducibility of the clusters $D_{r}(K)$ and the geometric measure $D_{s}(K)$. Last it is also possible 


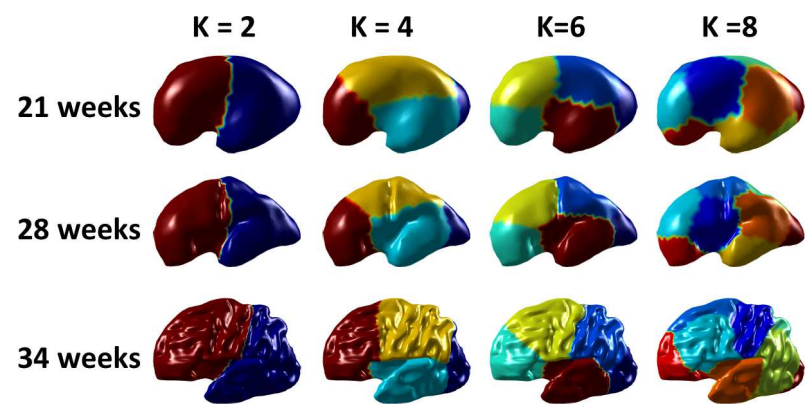

Fig. 1. Examples of parcellation of the left hemispheric cerebral surfaces for fetuses with GA of 21 (fist row), 28 (second row), and 34 (third row) weeks for $K=2,4,6$, and $K=10$.

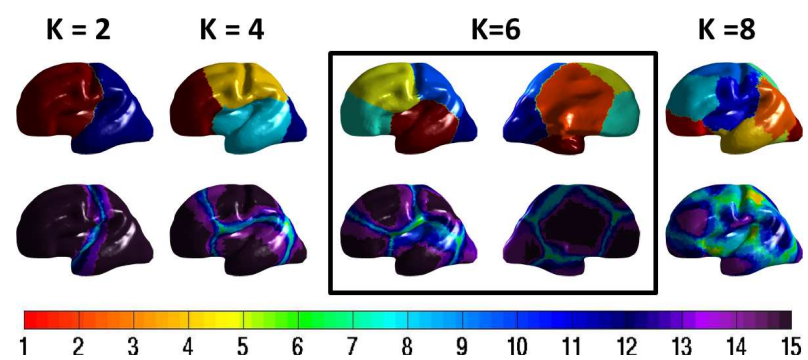

Fig. 2. Examples of most common parcel label (first row) and corresponding frequencies of occurrence (second row) in lateral view depicted for $K=2,4,6$, and $K=10$. For the case $K=6$, medial views are also depicted (fourth column).

to investigate the variability of the parcels for each $K$ thanks to the inter-subject distance matrix $\left(\operatorname{Dist}_{2}\left(f_{p}, f_{q}\right)\right)_{p, q}$. By using a 1-dimensional isomapping [11], we are able to have a representation of the $N$ subjects on an axis and to correlate it with age.

\section{RESULT}

\subsection{Data and pre-processing}

In utero acquisitions were performed on a 1.5-T Siemens MRI system with a T2-weighted half-Fourier-acquisition singleshot turbo spin-echo sequence (HASTE). In all the acquisitions, three stacks of 2D slices were obtained on axial, coronal and sagittal planes with respect to the fetal brain with a $0.742 \times 0.742 \mathrm{~mm}^{2}$ in plane spatial resolution and a $3 \mathrm{~mm}$ slice thickness. Mothers were sedated during the scanning to reduce motion artifacts in the images. To further compensate for spontaneous fetal motion artifacts while obtaining isotropic high-resolution 3D volumes, stacks of acquired orthogonal 2D MRI slices were processed using the slice intersection reconstruction technique described in [12]. The spatial resolution of the reconstructed 3D MRI volumes is $0.75 \mathrm{x}$ $0.75 \times 0.75 \mathrm{~mm}^{3}$.

Among all the scannings, 15 fetuses with no apparent anatomical abnormalities were retrospectively selected based on radiological criteria from the fetal clinical database ac-
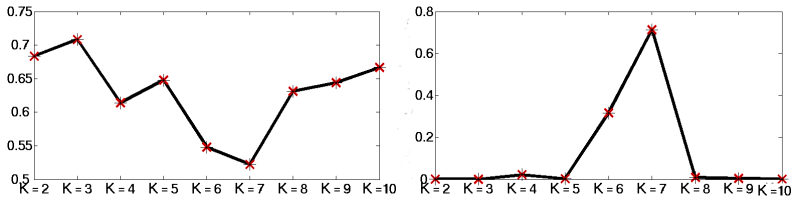

Fig. 4. The $r^{2}$ (left) and the p-values (right) of (a) age vs. distance, and (b) area vs distance for different values of $\mathrm{K}$.

quired in La Timone Hospital (Marseille, France), and used as the sample of this study. Gestational Age (GA) at the time of MRI acquisition varied from 21 to 34 weeks (mean age: 29.6 \pm 3.5 weeks). The developing cortex and the white matter zone in the left cerebral hemispheres of each fetuses included in this study were semi-automatically segmented in BrainVisa software ${ }^{1}$ to obtain a smooth triangulated mesh of the gray matter/white matter boundary. The CS and POS lines were then manually traced by an expert using the SurfPaint feature [13] of BrainVisa.

\subsection{Evaluation results}

We have applied our spectral clustering based parcellation approach to segment fetal left cerebral surfaces into $K$ patches $(K=2 \ldots 10)$.

Qualitative evaluation. In Fig. 1 are depicted examples of the parcellated left cortical surfaces for a few $K$ values, and a few fetuses spanning the gestational ages in the sample. The parcellation results appeared to be very well consistent in all cases, and especially for lower numbers of parcels $(K \leq 7)$. Segmentation results obtained for very young fetuses (e.g. 21 weeks) were visually comparable to the parcellation obtained for older fetuses (and thus more folded brain surfaces), despite the underlying surfaces in the former being smooth and with little morphological information, and despite the segmentation approach being fully unsupervised. These observations are confirmed in Fig. 2 where the per-vertex label of the most common parcel across all the $N=15$ surfaces is depicted along with its frequency of occurrence. Notice that in many locations the frequency of occurrence is close to its maximum value $(N)$, especially for low values of $K$, which shows a good agreement of the parcellation across subjects.

Quantitative evaluation. In Fig. 3 are shown the results of the quantitative evaluation in terms of parcellation's reproducibility $D_{r}(K)$, and in terms of the geometric measure $D_{s}(K)$. The $D_{r}(K)$ measure (see Fig. 3.(a)) increased with $K$. Furthermore, our quantitative results indicated that the measure $D_{s}(K)$ (and both its two components calculated from the CS and the POS lines) had a minimum value for $K=6$ (see Fig. 3.(b)). Notice also in Fig. 3.(c) that the value $K=6$ can be seen as a satisfying trade-off between the two measures $D_{r}(K)$ and $D_{s}(K)$.

\footnotetext{
${ }^{1}$ http://brainvisa.info/index_f.html
} 


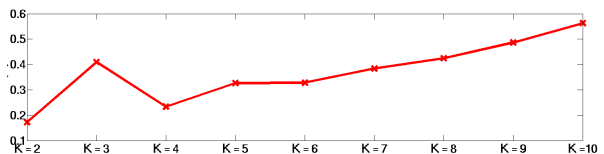

(a)

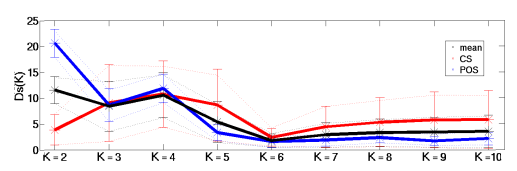

(b)

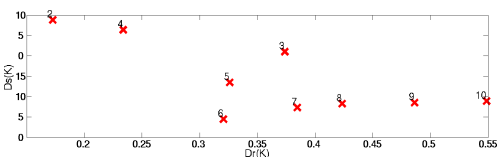

(c)

Fig. 3. The $D_{r}(K)$ and $D_{S}(K)$ measures are depicted in panels (a) and (b) respectively. In panel (c) is depicted the plot of the $D_{s}(K)$ versus $D_{r}(K)$.

\subsection{Effects of gestational age}

Next we studied the relationship between the remaining variability of parcels and the gestational age for each $K$. We found that the variability in the 1-dimensional isomapping of the inter-subject distance matrix (2) explained by the age factor was smaller for $K=6$ and $K=7$ as compared to the other studied $K$ values (see Fig. 4). In other worlds this result seems to suggest that the reproducibility of the cortical parcellation was less strongly related to the GA for $K=6$ and $K=7$ as compared to the other $K$ values. It is quite striking in Fig. 1 and Fig. 2 how the parcellation of the cortical surface obtained for $K=6$ well resembles the classical segmentation of the lateral cortical surface into frontal, parietal, temporal, occipital lobes, unless for the frontal lobe that were sub-segmented in its most frontal and posterior regions. For this reason, the use of $K=6$ clusters, which is somehow more 'optimal' than $K=7$ with respect to the $D_{s}(K)$ and $D_{r}(K)$ measures (see Sec. 3.2), should be preferred in the studies aiming at parcellating fetal cortical surfaces into lobes.

\section{DISCUSSION AND CONCLUSION}

In this work we have demonstrated the use of our previous algorithm [3] for spectral-based parcellation of cerebral hemispheres to a data-set of fetal MRI. Results obtained with our intrinsic and unsupervised approach confirmed the stability of the obtained parcellation for fetal data with varying gestational ages, and thus with varying levels of cortical gyrification. We quantitatively assess the anatomical relevance of our parcellation algorithm by measuring, within each subject, the alignment of parcel boundaries with two early folds. Interestingly, parcels obtained in the case $K=6$ ('optimal' with respect to a measure of parcellation reproducibility and another measure more related to geometrical features) could be qualitatively related to the brain lobes. In addition, our experiments confirmed the important relationship found in [3] between Fourier modes of a brain surface and anatomical features that are relevant for lobar segmentation.

\section{REFERENCES}

[1] X. Cheng, J. Wilm, S. Seshamani, M. Fogtmann, C. Kroenke, and C. Studholme, "Adapting parcellation schemes to study fetal brain connectivity in serial imaging studies," in EMBC'2013. IEEE, 2013, pp. 73-76.
[2] Zhang Y., Fan L., Yu C., and Jiang T., "Anatomical parcellation of human brain using structural covariance," in ISBI'2014, IEEE, 2014, pp. 858-861.

[3] J. Lefèvre, G. Auzias, and D. Germanaud, "Brain lobes revealed by spectral clustering," in ICPR, 2014.

[4] U. Von Luxburg, "A tutorial on spectral clustering," Stat Comput, vol. 17, no. 4, pp. 395-416, 2007.

[5] Ng A. Y, M. Jordan, and Y. Weiss, "On spectral clustering: Analysis and an algorithm," Adv Neural Inf Process Syst, vol. 2, pp. 849-856, 2002.

[6] M. Desbrun, M. Meyer, P. Schröder, and A. H. Barr, "Implicit fairing of irregular meshes using diffusion and curvature flow," in SIGGRAPH 99, 1999, pp. 317-324.

[7] M. Berger, A panoramic view of Riemannian geometry, Springer Verlag, 2003.

[8] G. Auzias, J. Lefèvre, A. Le Troter, C. Fisher, M. Perrot, J. Régis, and O. Coulon, "Model-driven harmonic parameterization of the cortical surface: HIP-HOP," IEEE TMI, vol. 32, no. 5, pp. 873-887, 2013.

[9] J. Munkres, "Algorithms for the assignment and transportation problems," J Soc Ind Appl Math, vol. 5, no. 1, pp. 32-38, 1957.

[10] J. Dubois, M. Benders, A. Cachia, F. Lazeyras, R. H.V. Leuchter, S. V. Sizonenko, C. Borradori-Tolsa, J. F. Mangin, and P. S. Hüppi, "Mapping the early cortical folding process in the preterm newborn brain," Cereb Cortex, vol. 18, no. 6, pp. 1444-1454, 2008.

[11] L. J. P. van der Maaten and G. E. Hinton, "Visualizing high-dimensional data using t-SNE," JMLR, pp. 25792605, 2008.

[12] F. Rousseau, E. Oubel, J. Pontabry, M. Schweitzer, C. Studholme, M. Koob, and J. L. Dietemann, "Btk: An open-source toolkit for fetal brain mr image processing," Comput Methods Programs Biomed, vol. 109, no. 1, pp. 65-73, 2013.

[13] Le Troter A., D. Riviere, and Coulon O., "An interactive sulcal fundi editor in brainvisa," in $O H B M, 2011$. 